\title{
Hereditary Male Breast Carcinoma
}

National Cancer Institute

\section{Source}

National Cancer Institute. Hereditary Male Breast Carcinoma. NCI Thesaurus. Code C36106.

Breast carcinoma that has developed in male relatives of patients with history of breast carcinoma. 